\title{
Hierarchical Assembly of Nucleic Acid/Coiled-Coil Peptide Nanostructures
}

\author{
Alex Buchberger, ${ }^{\dagger}{ }^{\ddagger}$ Chad R. Simmons, ${ }^{\ddagger \odot}$ Nour Eddine Fahmi, ${ }^{\ddagger}$ Ronit Freeman, ${ }^{\S}$ \\ and Nicholas Stephanopoulos $*,+,+$ (i) \\ ${ }^{\dagger}$ School of Molecular Sciences, Arizona State University, Tempe, Arizona 85287, United States \\ ${ }^{\ddagger}$ Center for Molecular Design and Biomimetics, The Biodesign Institute, Arizona State University, Tempe, Arizona 85287, United \\ States \\ ${ }^{\S}$ Department of Applied Physical Sciences, University of North Carolina, Chapel Hill, North Carolina 27514, United States
}

\section{Supporting Information}

ABSTRACT: DNA and peptides are two of the most commonly used biomolecules for building self-assembling materials, but few examples exist of hybrid nanostructures that contain both components. Here we report the modification of two peptides that comprise a coiled-coil heterodimer pair with unique DNA handles in order to link DNA origami nanostructures bearing complementary strands into micrometer-long one-dimensional arrays. We probed the effect of number of coils on self-assembly and demonstrated the formation of structures through multiple routes: one-pot assembly, formation of dimers and trimers and an alternating copolymer of two different origami structures, and stepwise assembly of purified structures with coiled-coil conjugates. Our results demonstrate the successful merging of two distinct self-assembly modes to create hybrid bionanomaterials expected to have a range of potential applications in the future.

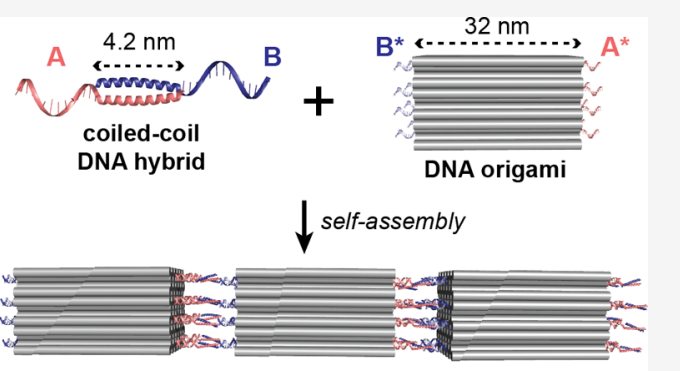

1D nanofiber driven by multivalent coiled-coil assembly

\section{INTRODUCTION}

The self-assembly of programmable materials using biological molecules is a central goal of nanotechnology, with potential applications in regenerative medicine, targeted drug delivery, energy transfer materials, fundamental biology, and materials science. DNA is one of the most useful building blocks for selfassembled materials due to the programmable nature of Watson-Crick pairing. In the past three decades, the field of DNA nanotechnology has demonstrated a wealth of complex, anisotropic, and highly functional materials, as exemplified by DNA origami, ${ }^{1-3}$ tile-based approaches, ${ }^{4-7}$ and nanostructures with programmable and dynamic properties. ${ }^{8,9}$ Polypeptides, by contrast, have the advantage of greater chemical diversity made available through the 20 canonical amino acids, and a broad range of synthetic noncanonical amino acids if solidphase synthesis is used. Peptides also possess multiple structural motifs (e.g., $\alpha$-helices, $\beta$-sheets) and self-assembly modes (e.g., coiled-coils, collagen triple helices) that impart unique functional and mechanical properties. While several examples exist of multivalent recombinant proteins covalently modified with DNA to form cages, ${ }^{10}$ nanofibers, ${ }^{11,12}$ and threedimensional crystals, ${ }^{13}$ the chemical coupling of synthetic selfassembled peptides with DNA has only recently been demonstrated. The Ke and Conticello laboratories reported the first example of linking DNA origami nanostructures with a synthetic peptide self-assembly motif, using collagen-mimetic triple helices fused with a cationic domain to electrostatically associate with the negatively charged oligonucleotide structures. $^{14}$ The Stupp lab pioneered the modification of selfassembled peptide amphiphile nanofibers with DNA to drive their hierarchical, and reversible, assembly into bundles through DNA hybridization, and demonstrated that these superstructures could control cell behavior. ${ }^{15}$ The Freeman lab extended this principle to short self-assembling Fmocdipeptides grafted to oligonucleotide handles to enable the reversible modulation of hierarchical structures. ${ }^{16}$

One particularly attractive motif for hybrid nanomaterials is the coiled-coil assembly between two $\alpha$-helical peptides, driven by a combination of hydrophobic and charge-charge interactions. ${ }^{17} \mathrm{~A}$ heterodimeric coiled-coil resembles a DNA duplex in many respects, namely, sequence-specific formation of a linear self-assembled structure with well-defined structural and physical properties. However, coiled-coils have a number of properties that diverge from DNA, which makes them both more difficult to work with, but also promising for additional applications. Unlike the standard B-form DNA duplex, coiledcoils can form in both parallel and antiparallel forms, adopt a range of homo- and hetero-oligomeric states, and complex nanostructures made of coils possess high stability in biologically relevant buffers. Coiled-coils allow the positioning of components closer to one another compared with DNA; for

Received: October 16, 2019

Published: December 10, 2019 
A

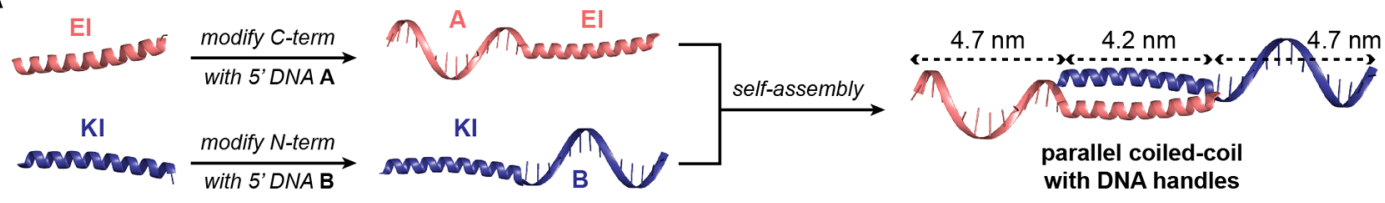

B

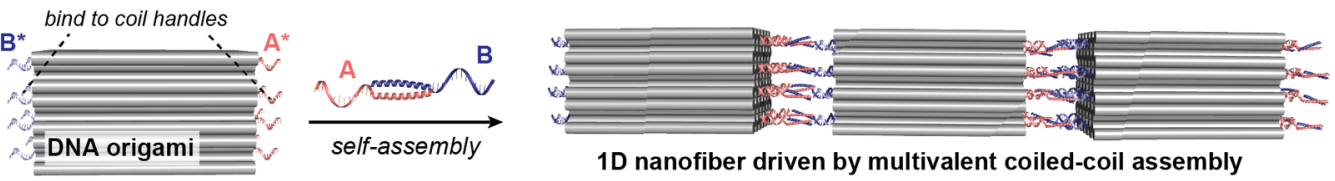

C $\begin{array}{ll}\text { EI: Ac-EIAALEKEIAALEKENAALEWEIAALEK(azK)-NH } & \mathrm{KI}: \text { Ac-(azK)KIAALKEKIAALKEKNAALKWKIAALKE-NH } \\ 2\end{array}$

D
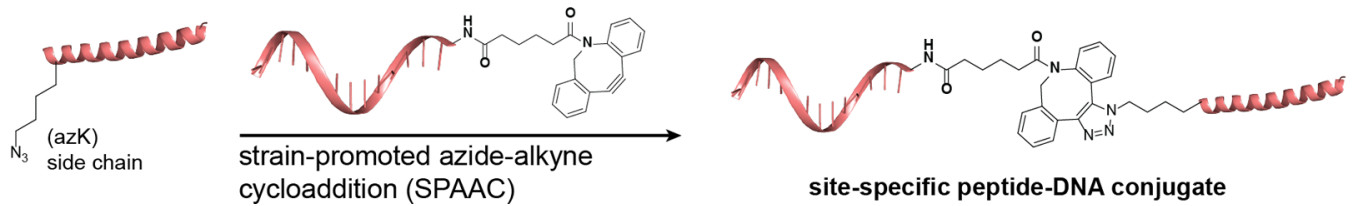

site-specific peptide-DNA conjugate

Figure 1. Overview of strategy for assembling DNA origami with coiled-coils. (A) Site-specific functionalization of two peptides comprising a coiled-coil with DNA. Mixing the two peptide-DNA conjugates yields a self-assembling coiled-coil with two orthogonal oligonucleotide handles. (B) Assembling a DNA origami structure (e.g., a cuboid) with multiple ssDNA handles into 1D nanofibers driven by the coiled-coil bearing complementary handles. $(C)$ Amino acid sequences $(\mathrm{N} \rightarrow \mathrm{C}$ ) of the four peptides used in this study; (azK) indicates azidolysine. (D) Strainpromoted alkyne-azide cycloaddition (SPAAC) conjugation between the side chain of (azK) and the dibenzocyclooctyne (DBCO) moiety on DNA to yield a peptide-DNA conjugate.

example, an $\alpha$-helix has a pitch of $0.54 \mathrm{~nm}$, compared with 3.4 $\mathrm{nm}$ for B-form DNA. Furthermore, the ability to incorporate synthetic amino acids into the constituent peptides will also allow coils to be used as molecular scaffolds that can position chemical groups (e.g., energy transfer dyes, nanoparticles, drugs) in close proximity, or with a higher density, than modifying origami staple strands alone. We also note that coiled-coil DNA conjugates enable integration of recombinant proteins through genetic fusion of one of the two peptide partners, and may enhance the stability of hybrid nanomaterials by not requiring supraphysiological counterion concentrations. $^{18}$

As a result of their unique advantages, coiled-coils have been used to generate a wide range of bionanomaterials, including: extended one-dimensional nanofibers, ${ }^{19,20}$ hydrogels, ${ }^{21}$ hollow cages, $^{22}$ and wireframe structures from the intramolecular folding of a long polypeptide containing multiple orthogonal coiled-coil pairs. ${ }^{23}$ The first example of DNA-modified coiledcoils was reported by the Wengel and Jensen laboratories, and used DNA triplex formation to template and stabilize a homotrimeric peptide interaction. ${ }^{24}$ Very recently, the Woolfson and Turberfield laboratories demonstrated the use of DNA origami structures to determine the effect of multivalency on peptide binding constants. ${ }^{25}$ This elegant report used the origami as both a scaffold and visualization marker to simultaneously control the number of coiled-coils (from one to three) and to quantify their interactions (via origami dimer formation) that would be difficult to capture otherwise. In parallel we envisioned an alternate approach, whereby a coiled-coil peptide pair modified with DNA handles could serve as a modular supramolecular building block and assemble DNA structures into higher order assemblies. The coiled-coil would impart all the properties of the peptides (e.g., chemical and structural diversity), whereas the DNA would serve as a programmable building block to dictate the dimensions and properties of the final structures, as well as the multivalency and nanoscale spacing of the peptides.

Here, we demonstrate the use of coiled-coil peptide-DNA conjugates to link together DNA origami structures bearing complementary strands and to generate one-dimensional (1D) supramolecular polymers. Our design relies on chemically modifying each peptide of a heterodimer coiled-coil pair with a short DNA strand, resulting in a self-assembled core with two addressable handles (Figure 1A). These handles are then used to link together a DNA origami cuboid bearing a tunable number of complementary handles at its two ends. We demonstrate the formation of nanofibers consisting of rigid origami units linked by multiple coiled-coil pairs (Figure 1B), with the longest fibers consisting of almost 80 origami units and exceeding three micrometers in length. By spatially confining multiple coiled-coils on the DNA cuboids, we effectively create a polypeptide-based interface between origami structures, akin to protein-protein interactions. We probed the effect of peptide multivalency on the efficiency of nanofiber assembly, and explored multiple self-assembly pathways: one-pot formation of both DNA and coiled-coil self-assembly, hierarchical formation of dimer and trimer structures as well as alternating copolymers, and linking of purified origami with coiled-coils. Taken together, our results show that peptide-DNA conjugates can be used to construct hierarchical nanomaterials that integrate more than one selfassembly "mode" to create a biomolecular structure in a controlled and programmable fashion.

\section{RESULTS AND DISCUSSION}

Synthesis and Characterization of Peptide-DNA Conjugates. For the coiled-coil, we selected a pair of peptides developed by Aili and co-workers. ${ }^{26}$ One peptide (termed EI) 


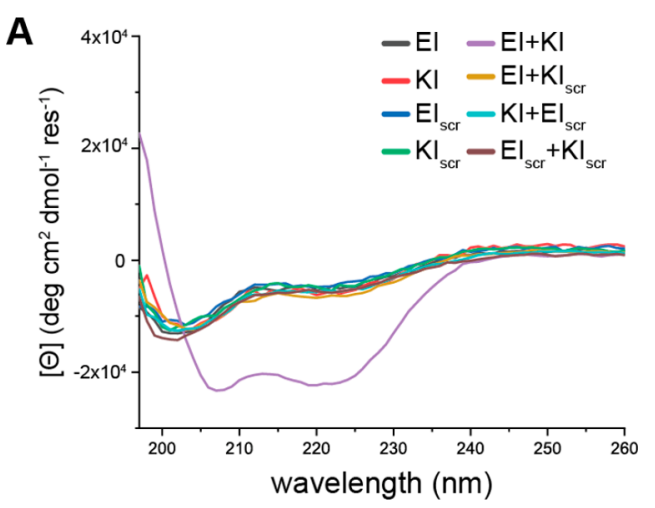

D

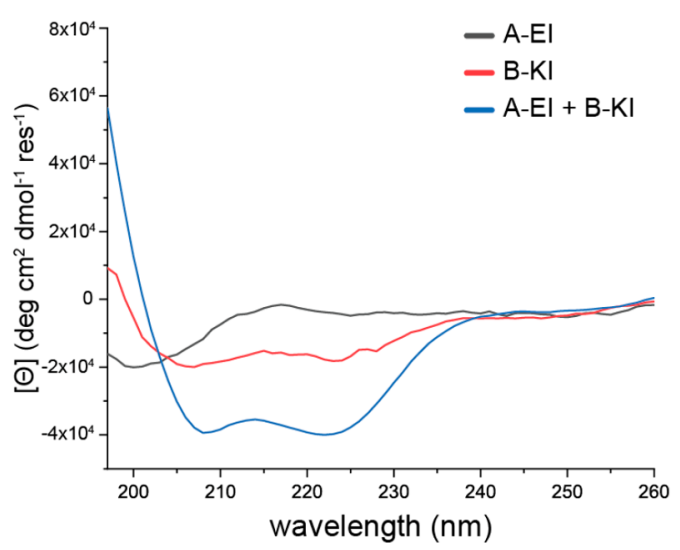

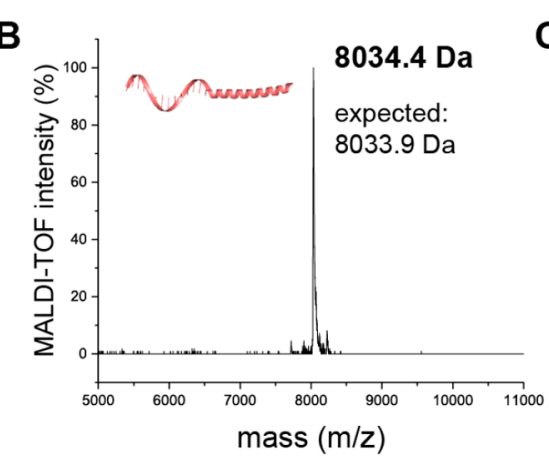

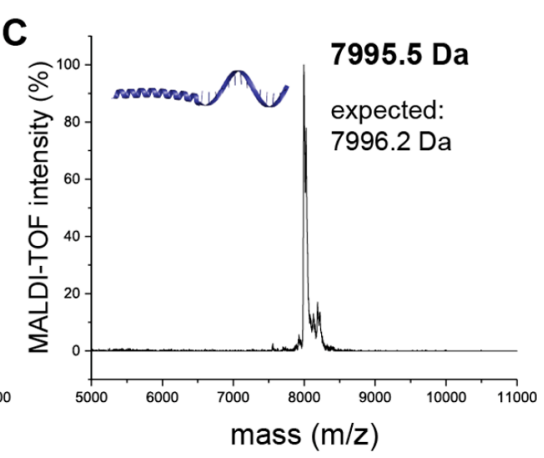

E

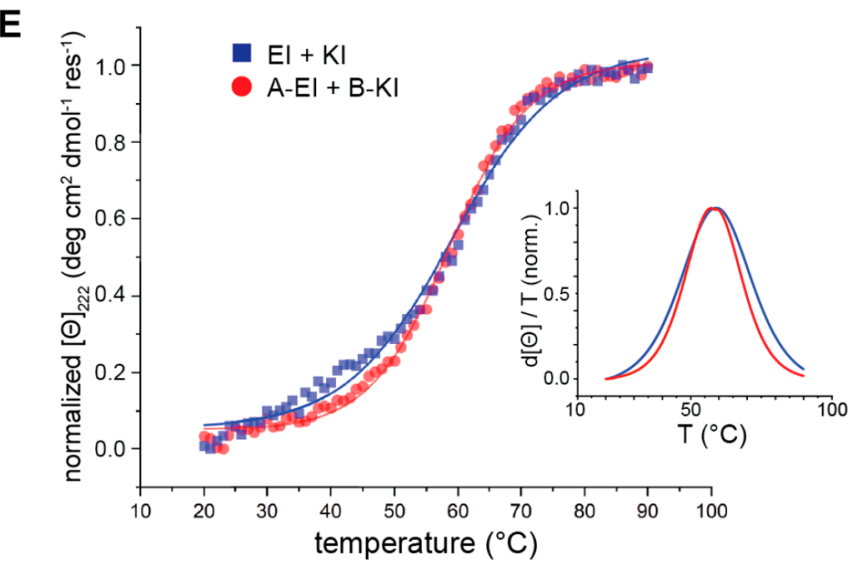

Figure 2. Characterization of peptide-DNA conjugates. (A) Circular dichroism (CD) spectroscopy of the peptides or peptide combinations indicated. Only the EI + KI sample shows a strong coiled-coil signal. (B,C) MALDI-TOF mass spectra for A-EI (B) and B-KI (C). (D) CD analysis of the peptide-DNA conjugates indicating coiled-coil formation between the peptides. (E) Temperature-dependent CD analysis (222 nm) of the peptides and peptide-DNA conjugates to determine the melting temperature of the coiled-coil interaction. Inset: first derivative of CD melting curve.

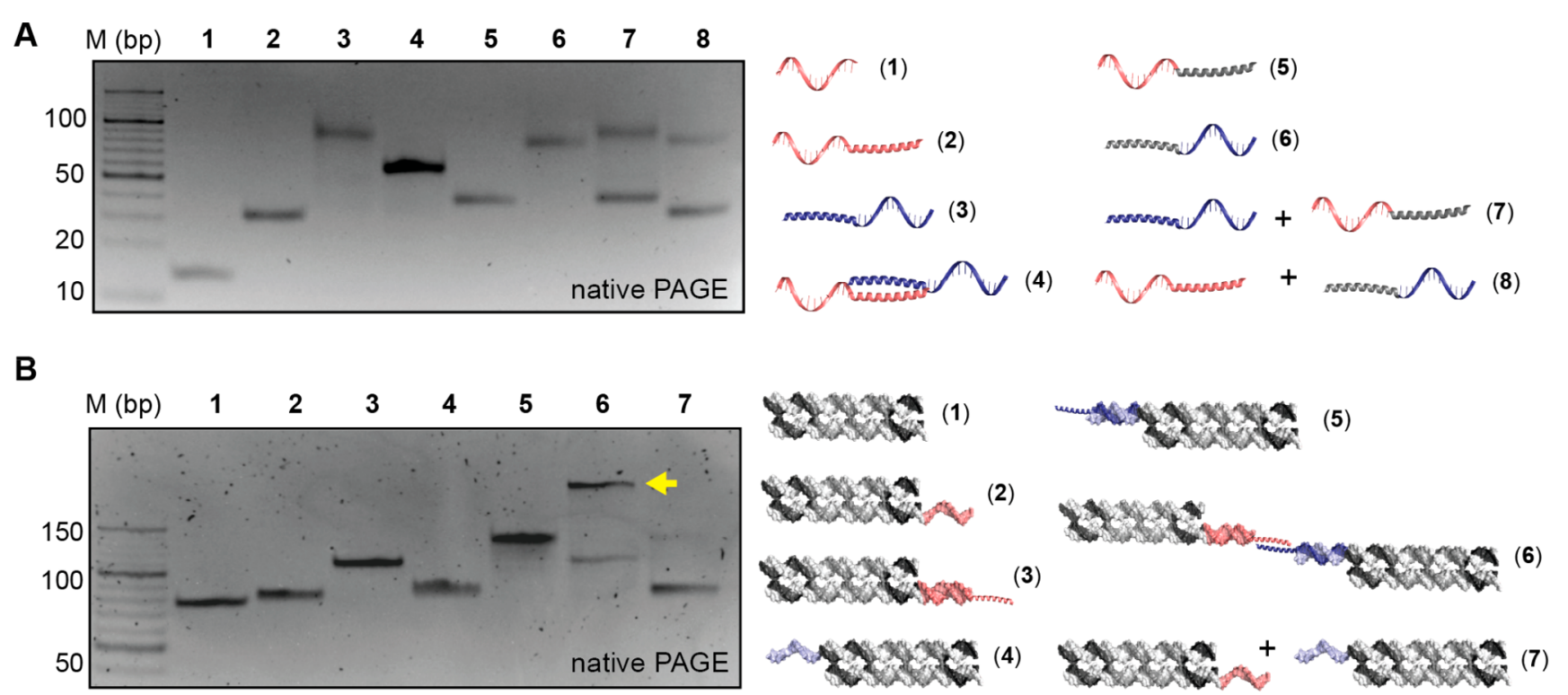

Figure 3. Polyacrylamide gel electrophoresis (PAGE) analysis of coiled-coil assembly. For both gels, the cartoons with numbers in parentheses indicate the major species in each lane. (A) Native PAGE of peptide-DNA conjugates. DNA strands are depicted as single-stranded, but had their complement added prior to running the gel in order to generate dsDNA and enhance staining. Lane M: dsDNA ladder (bp); 1: DNA sequence A; 2: A-EI conjugate; 3: B-KI conjugate; 4: A-EI + B-KI; 5: A-EI ${ }_{\text {scr }}$ 6: B-KI ${ }_{\text {scr }}$ 7: B-KI + B-KI $\mathrm{scr}_{\mathrm{B}-\mathrm{KI}}+\mathrm{A}-\mathrm{EI} \mathrm{scr}_{\text {; }}$ A-EI + B-KI $\mathrm{scr}$ (B) Native PAGE of double-crossover (DX) tiles bearing coiled-coil peptides. Lane M: dsDNA ladder (bp); 1: DX tile; 2: DX tile with handle extension A*; 3: DX tile with A-EI hybridized; 4: DX tile with handle extension B*; 5: DX tile with B-KI hybridized; 6: assembly of DX tiles bearing A-EI and B-KI; 7: equimolar mixture of tiles bearing handles, but without peptides attached. The yellow arrow in lane 6 indicates the DX tile dimer; comparison with the lower band indicates a dimerization efficiency of $\sim 75 \%$. 

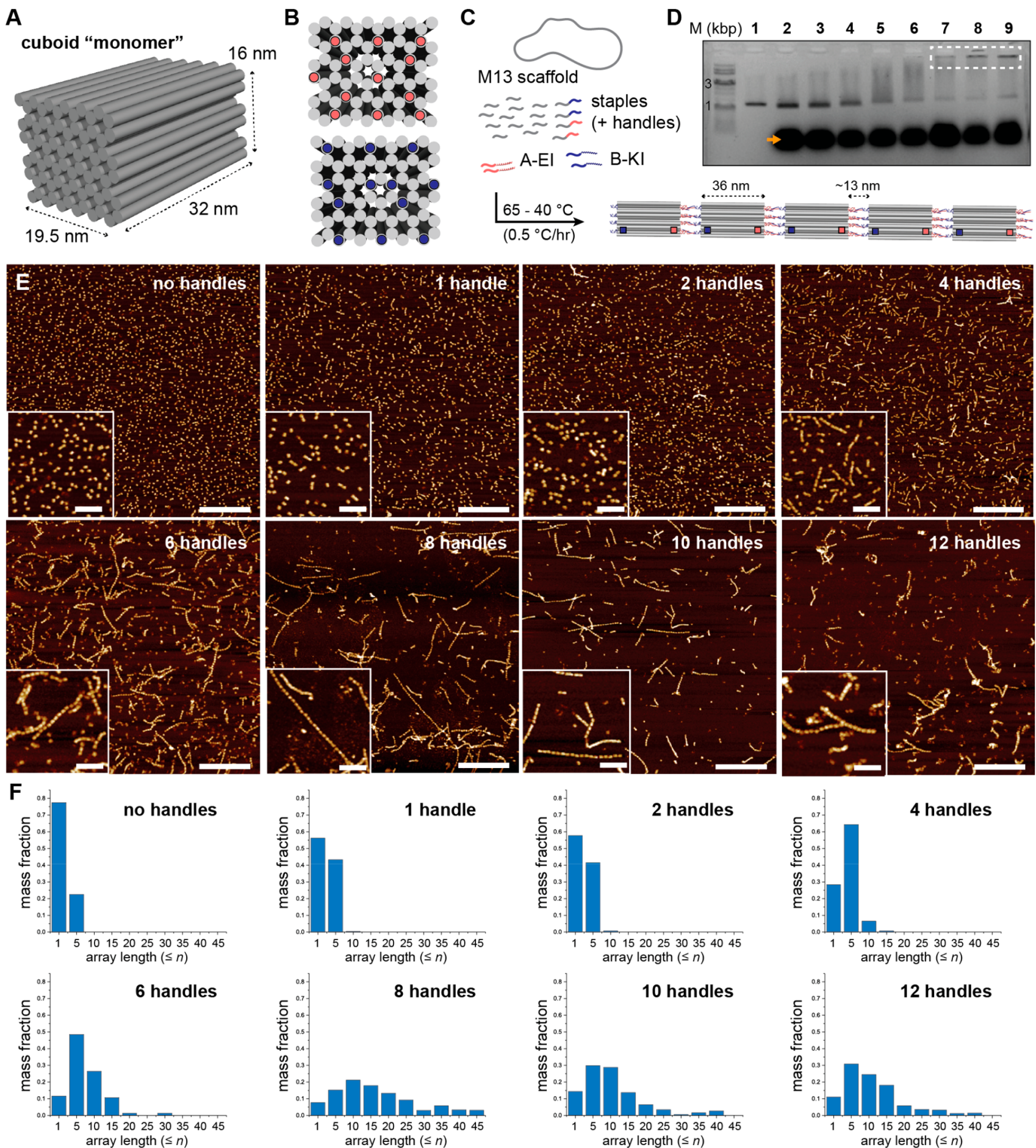

Figure 4. One-pot assembly of DNA origami nanofibers using coiled-coils. (A) Design and dimensions of origami cuboid "monomer" used. (B) Location of 12 possible handles for A-EI (pink) and B-KI (blue). (C) Protocol for one-pot assembly of fibers. Pink and blue squares on the origami indicate the location of handles $\mathrm{A}^{*}$ and $\mathrm{B}^{*}$, respectively. (D) Agarose gel electrophoresis (AGE) analysis of one-pot assembly. Lane M: dsDNA ladder (bp); 1: M13 scaffold strand; 2: cuboid monomer with no handles for peptides; 3-9: cuboids with 1, 2, 4, 6, 8, 10, and 12 handles, respectively. The orange arrow indicates excess staples and peptide-DNA conjugates. (E) AFM images of indicated samples. Scale bars: $1 \mu \mathrm{m}$ (zoom-out), $250 \mathrm{~nm}$ (insets). (F) Histograms of mass fraction of origami for each array length for indicated samples. Numbers correspond to arrays with length equal to that between the value of the previous bin and indicated number, e.g., " 25 " indicates arrays $21-25$ cuboids in length.

is net anionic $(-5)$, while the other (termed $\mathrm{KI})$ is net cationic $(+5$, Figure $1 \mathrm{C})$, and the two form a parallel heterodimer $\left(\sim 4.2 \mathrm{~nm}\right.$ in length) with a $K_{\mathrm{d}}$ below $0.1 \mathrm{nM}$. We reasoned that such a high affinity would be useful in order to link DNA origami structures, which are typically assembled at lownanomolar concentrations, with additional enhancement due to multivalency. The peptides were obtained by solid-phase synthesis with Fmoc-protected amino acids, and we incorporated an azidolysine $(\mathrm{azK})$ residue at the $\mathrm{N}$-terminus of $\mathrm{KI}$ and the C-terminus of EI to allow for DNA conjugation via strainpromoted azide-alkyne cycloaddition (SPAAC) chemistry $^{27}$ (Figure 1D). For the details of peptide synthesis, purification, 
and characterization, see Supporting Information section S2. When mixed in an equimolar ratio in phosphate buffered saline (PBS), EI and KI yielded a corresponding coiled-coil signal by circular dichroism (CD) with the characteristic peaks at 208 and $222 \mathrm{~nm}$ as seen by Aili et al. ${ }^{26}$ (Figure $2 \mathrm{~A}$ ). In order to functionalize each peptide, we synthesized two 14-nt DNA handles (termed A and B, see Supporting Information section S3 for strand sequences) modified with dibenzocyclooctyne (DBCO) at the $5^{\prime}$ end through an amine linker. The two peptides were coupled via SPAAC to the DNA handles to generate two peptide-DNA conjugates A-EI and B-KI. Each peptide-DNA conjugate was purified by reverse phase HPLC and characterized by MALDI-TOF MS (Figure 2B,C), HPLC (Figure S2), and native polyacrylamide gel electrophoresis (PAGE, vide infra). We note that the cationic KI peptide tended to aggregate with the DNA-DBCO at higher concentrations due to electrostatic effects, but we were able to synthesize it successfully by carefully controlling the reaction conditions (see section S3). As controls, we synthesized two peptides with the same amino acid composition as EI and KI, but with the residues scrambled in order to abolish the heptad repeat pattern characteristic of coiled-coil assembly (Figure 1C). These peptides, which we term $\mathrm{EI}_{\text {scr }}$ and $\mathrm{KI}_{\text {scr }}$, did not yield a coiled-coil with $\mathrm{KI}$ and $\mathrm{EI}$, respectively, as monitored by $\mathrm{CD}$ (Figure $2 \mathrm{~A}$ ). We conjugated the scrambled peptides to the same two DNA handles to yield $\mathrm{A}-\mathrm{EI}_{\text {scr }}$ and $\mathrm{B}-\mathrm{KI}_{\text {scr }}$ (see section S3 for characterization).

Probing Coiled-Coil Formation with Peptide-DNA Conjugates. We first investigated whether the A-EI and B-KI conjugates could still self-assemble via CD (Figure 2D). At 10 $\mu \mathrm{M}$, the two peptide-DNA conjugates gave a signal with minima at 208 and $222 \mathrm{~nm}$ that was virtually indistinguishable from that of the individual peptides (Figure 2A), supporting the formation of a coiled-coil. Interestingly, the melting temperature of this A-EI/B-KI assembly was $58.5{ }^{\circ} \mathrm{C}$, which was indistinguishable from that of the $\mathrm{EI} / \mathrm{KI}$ peptide pair at that concentration (Figure 2E), suggesting that the DNA handles do not perturb coiled-coil stability. We also probed the coiled-coil assembly by native PAGE (Figure 3A). Compared with the unmodified 14-nt A strand (lane 1), the A-EI conjugate was shifted to a higher retention time due to its increased size (lane 2). The B-KI conjugate, by contrast, showed significantly reduced mobility by native PAGE (lane 3 ) due to the reduction in net negative charge resulting from the cationic peptide. We also note that the presence of a single band in lanes 1 and 2 further confirms the purity of the individual peptide-DNA conjugates. Combining A-EI and B$\mathrm{KI}$ in an equimolar ratio ( $1 \mu \mathrm{M}$ each) and briefly annealing them in 1xTAE- $\mathrm{Mg}^{2+}$ buffer resulted in a new band with a retention time between the two individual peptides, due to a balancing of increased size and increased negative charge (lane 4). To confirm that this band was due to the coiled-coil assembly, as opposed to nonspecific electrostatic adsorption, we used the peptide-DNA conjugates with scrambled sequences. Both $\mathrm{A}-\mathrm{EI}_{\mathrm{scr}}$ and $\mathrm{B}-\mathrm{KI}_{\mathrm{scr}}$ showed single bands by native PAGE (lanes 5 and 6), at roughly the same retention times as the unscrambled conjugates. Equimolar mixtures of A$\mathrm{EI}$ and $\mathrm{B}-\mathrm{KI}_{\mathrm{scr}}$ (lane 7) and $\mathrm{B}-\mathrm{KI}$ and $\mathrm{A}-\mathrm{EI}_{\text {scr }}$ (lane 8), however, showed only the bands for the individual peptide-DNA conjugates, with no shifted bands due to self-assembly. We also performed control experiments to confirm that the coiled-coil assembly of A-EI and B-KI could be abolished by the addition of excess free peptide to out-compete the desired interaction
(Figure S4). From these results, we concluded that the peptide-DNA conjugates efficiently formed a coiled-coil, and that this effect was due to the specific peptide sequences used.

In order to determine whether the coiled-coil interaction could assemble a model DNA nanostructure, we investigated the dimerization of double-crossover ("DX") tiles (Figure 3B, and Section S5). We designed two tiles with single-stranded extensions $\mathrm{A}^{*}$ and $\mathrm{B}^{*}$, complementary to $\mathrm{A}$ and $\mathrm{B}$. Native PAGE analysis of a DX tile with no handles (lane 1), the tiles with handles $\mathrm{A}^{*}$ and $\mathrm{B}^{*}$ (lanes 2 and 4 ), and tiles annealed with $\mathrm{A}-\mathrm{EI}$ and $\mathrm{B}-\mathrm{KI}$ to incorporate the individual peptides (lanes 3 and 5) showed clear single bands for the structures. We once again note that the cationic KI peptide especially decreased the mobility of the tile due to its charge. An equimolar mixture of the two tiles $(250 \mathrm{nM})$ resulted in a shifted band (lane 6, yellow arrow) that we attribute to a DX tile heterodimer. Comparing the band intensity to the unshifted band, we estimate the dimerization efficiency of $\sim 75 \%$ at this concentration and with a single coiled-coil interaction. Finally, a mixture of tiles bearing handles $A^{*}$ and $B^{*}$, but lacking peptides, showed no such shift (lane 7), supporting the role of coiled-coil formation. However, to assemble larger structures like origami (which are formed at much lower concentrations, typically $1-10 \mathrm{nM}$ ), we reasoned that it would be critical to incorporate multiple coiled-coil interactions.

One-Dimensional DNA Origami Arrays Using CoiledCoil Interactions. As our nanostructure "monomer" for creating $1 \mathrm{D}$ arrays, we selected a rectangular DNA origami cuboid reported by Walther and co-workers. ${ }^{28}$ This cuboid is rigid and well-defined, readily distinguishable by atomic force microscopy (AFM) and transmission electron microscopy (TEM), and allows for the controlled placement of multiple coiled-coil peptides. The cuboid has dimensions of $32 \times 19.5$ $\times 16 \mathrm{~nm}$ (Figure 4A), and was assembled through standard DNA origami thermal annealing of the M13 scaffold with a 10fold excess of staple strands in 1xTAE- $\mathrm{Mg}^{2+}$ buffer (for origami design, synthesis, and characterization see Section S6). We introduced between 1 and 12 single stranded handles with sequence $A^{*}$ and $B^{*}$ to the two ends of the cuboid (the $16 \times$ $19.5 \mathrm{~nm}$ surface) by extending the corresponding staples, allowing us to tune the number of coiled-coil interactions that would drive origami assembly (Figures 4B and S6). To induce nanofiber formation, we mixed the M13 scaffold strand, a 10fold excess of staples, and the A-EI and B-KI conjugates at equimolar concentration to their complementary handles (e.g., 80 -fold vs the scaffold strand for the cuboids bearing 8 handles on each end). This mixture, which we term the "one pot" assembly (Figure 4C), was annealed from $65-40{ }^{\circ} \mathrm{C}$ over 2 days $\left(0.5{ }^{\circ} \mathrm{C} / \mathrm{h} \mathrm{ramp}\right)$. We also highlight that stopping the slow thermal ramp at $40{ }^{\circ} \mathrm{C}$ prevented the formation of undesired and nonspecific aggregates due to base stacking of the origami blunt ends (vide infra). Despite the presence of 9fold excess unbound peptide-DNA conjugates-which might be expected to "cap" the peptides attached the origami, preventing growth into fibers-we reasoned that the multivalency should enable fiber formation ${ }^{28}$ due to exchange of peptide-DNA conjugates. Following annealing, we analyzed the samples by agarose gel electrophoresis (AGE), AFM, and negative-stain TEM.

By AGE, we observed a clear monomer band for the cuboid (Figure 4D, lane 2), which did not appear to shift when 1 or 2 handles for the coiled-coils were included in the annealing 

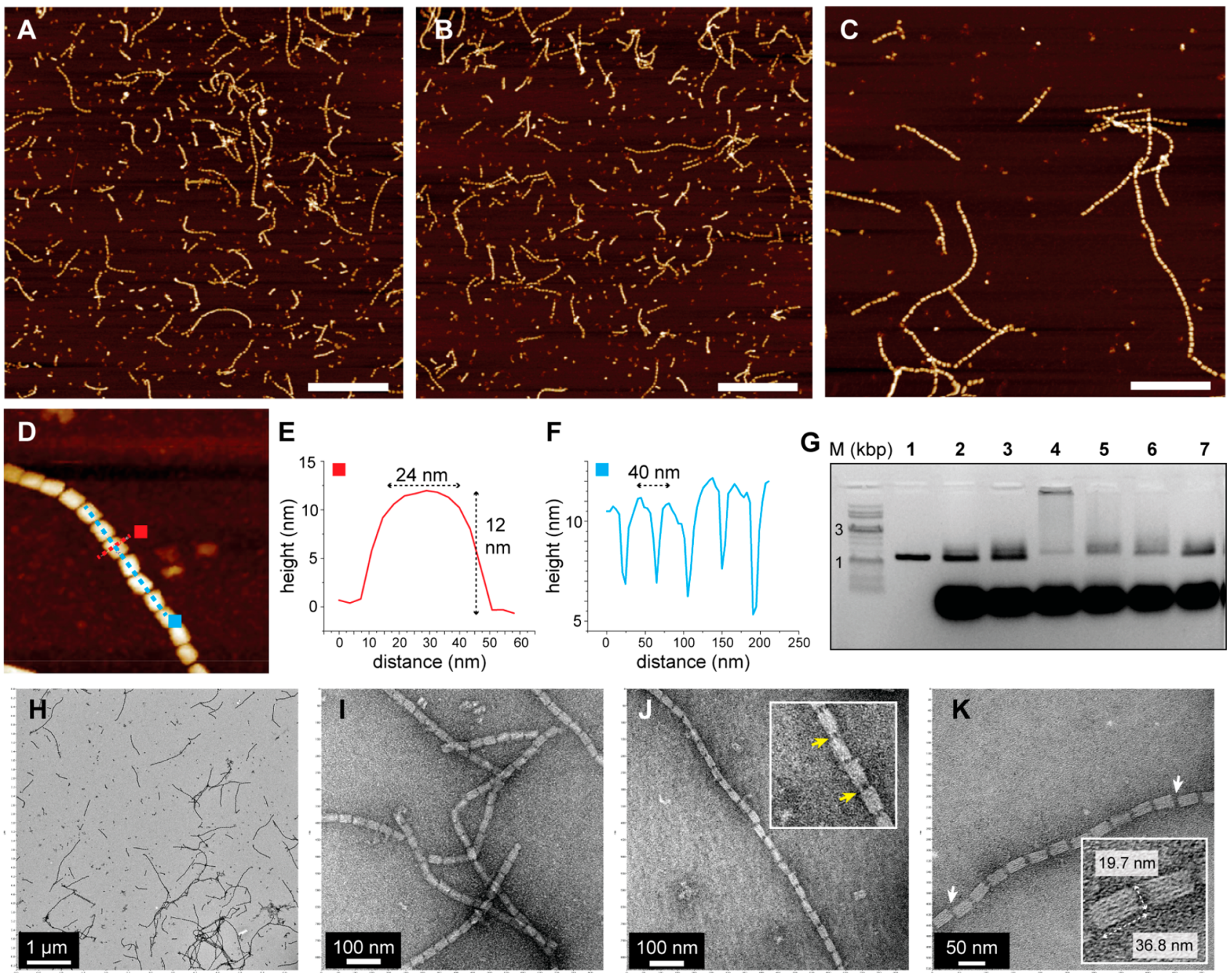

Figure 5. Analysis of one-pot cuboid assembly with 8 handles. (A-D) Additional AFM images of cuboid nanofibers assembled by coiled-coil interactions. Scale bars: $1 \mu \mathrm{m}$ (A,B), $660 \mathrm{~nm}$ (C). (E,F) Height profiles along the indicated red and blue dashed lines in (D). (G) AGE analysis of 8-handle assembly. Lane M: dsDNA ladder; 1: M13 scaffold; 2: cuboid monomer without handles; 3: cuboid with 8 handles $\left(A^{*}\right.$ and $\left.B^{*}\right)$ on each

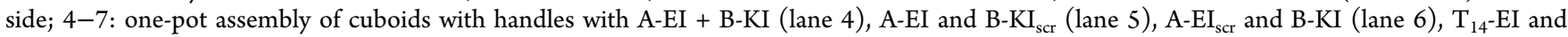
$\mathrm{T}_{14}$-KI (lane 7). ( $\left.\mathrm{H}-\mathrm{K}\right)$ Negative-stain TEM images of fibers at various degrees of magnification, scale bars as indicated. Inset in (J) shows direct visualization of links between cuboids (yellow arrows); inset in (K) shows the parallel helices and dimensions of the cuboids. The white arrows indicate partial, misaligned intercuboid interfaces.

mixture (lanes 3 and 4). The one-pot sample with 4 and 6 handles (lanes 5 and 6), by contrast, showed a broad, diffuse band across higher retention times that we attribute to a range of intermediate assembly state with two or more cuboids. The samples with 8, 10, and 12 handles (lanes 7-9), however, showed a strong band in the loading well (white dashed box), suggesting the formation of assemblies that were too large to enter the gel. We note that all lanes also had a thick, highmobility band (orange arrow) due to the excess staples and peptide-DNA conjugates. We imaged all samples by AFM (Figure 4E), and saw that samples with 1 or 2 handles were primarily monomers and dimers, whereas samples with 4 handles formed short oligomers. With 6 handles, we began to see a mixture of oligomers, small arrays, and a distinct population of longer arrays (>10 cuboids). The samples with 8 , 10 , and 12 handles, by contrast, exhibited a significant fraction of very long structures. We quantified these results by determining the number of cuboids per fiber via AFM. We calculated the "mass fraction," which we define as the fraction of total cuboids that comprise arrays of a given length, and plotted the histograms shown in Figure 4F (see Figures S8S17 for unbinned histograms that explicitly quantify every array length). These results parallel the AFM images: the majority of cuboids with no handles are monomers $(78 \%$, though we were conservative in excluding dimers, so this number may be higher), with the remaining $22 \%$ being dimers or other short oligomers (3-5 monomers) due to base stacking. For cuboids bearing 1 and 2 handles, the fraction of dimers and short oligomers increased to $43 \%$ and $41 \%$, respectively. With 4 handles, the majority of origami (64\%) are in oligomers 2-5 units long, with a small fraction (7\%) forming arrays $6-10$ or $11-15$ monomers. With 6 handles, the distribution shifted to even longer arrays, with a significant number of structures 6-10 and 11-15 monomers in length (26 and 10\%, respectively). However, the sample with 8 handles showed a broad distribution with the longest arrays, with a peak at 6-10 monomers (21\%), and a long tail encompassing arrays 11 monomers or more, including many structures 30-45 cuboids long. Interestingly, the samples with 10 and 12 handles did not have as many long arrays as the 
A

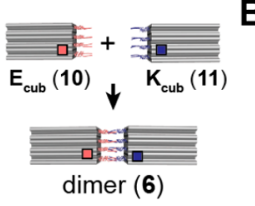

B

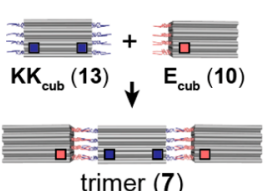

D

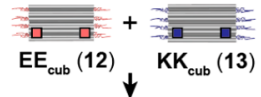

C

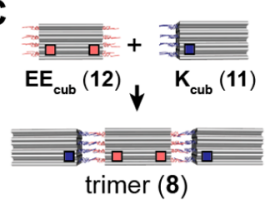

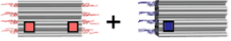

立
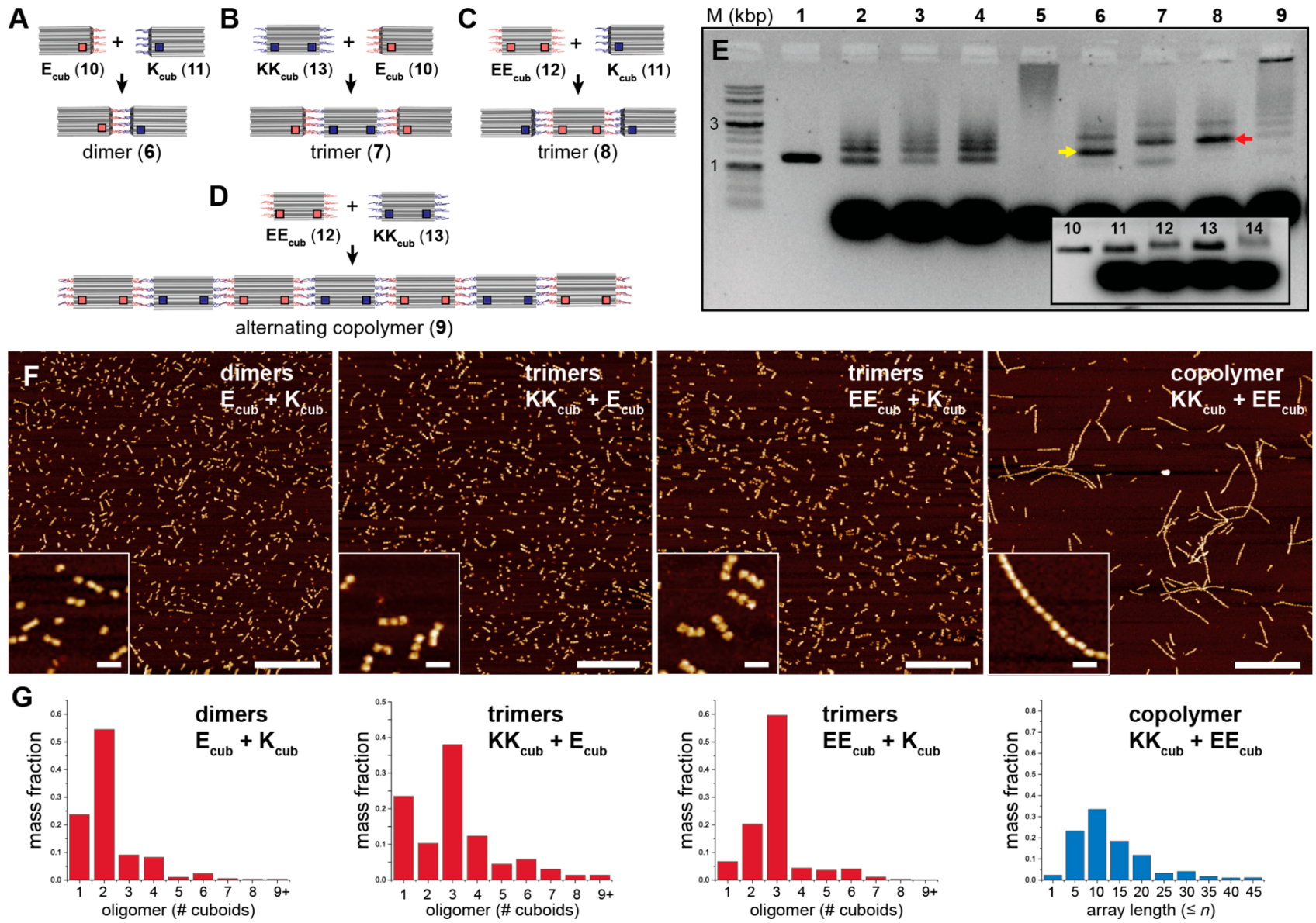

Figure 6. Hierarchical assembly of cuboids with peptide-modified faces. (A-D) Schematics of four assembly protocols probed by AGE. Pink and blue squares on the origami indicate the location of handles $\mathrm{A}^{*}$ and $\mathrm{B}^{*}$, respectively. (E) AGE analysis of hierarchical assembly, after second incubation at $32{ }^{\circ} \mathrm{C}$ for $15 \mathrm{~h}$. Lane M: dsDNA ladder (bp); 1: M13 scaffold; 2: $\mathrm{E}_{\mathrm{cub}} ; 3: \mathrm{K}_{\mathrm{cub}} ; 4: \mathrm{EE}_{\mathrm{cub}} ; 5: \mathrm{KK}_{\mathrm{cub}} ; 6: \mathrm{E}_{\mathrm{cub}}+\mathrm{K}_{\mathrm{cub}}$ (orange arrow: dimer band); 7: $\mathrm{E}_{\mathrm{cub}}+\mathrm{KK}_{\mathrm{cub}}$; 8: $\mathrm{EE}_{\mathrm{cub}}+\mathrm{K}_{\mathrm{cub}}$ (red arrow: trimer band); 9: $\mathrm{EE}_{\mathrm{cub}}+\mathrm{KK}_{\mathrm{cub}}$. Inset: AGE analysis of the individual cuboids prior to the second incubation. Lane 10: M13 scaffold; 11: $\mathrm{E}_{\mathrm{cub}} ; 12: \mathrm{K}_{\mathrm{cub}}$; 13: $\mathrm{EE}_{\mathrm{cub}}$; 14: $\mathrm{KK}_{\mathrm{cub}}$. (F) AFM images of samples indicated. Scale bars: $1 \mu \mathrm{m}$ (zoom-out), $100 \mathrm{~nm}$ (insets). (G) Histograms of mass fraction of origami for each oligomer or array length for indicated samples. The number for $\mathrm{EE}_{\mathrm{cub}}+\mathrm{KK}_{\mathrm{cub}}$ samples corresponds to arrays between previous bin and indicate length, e.g., " 25 " indicates arrays $21-25$ cuboids in length.

sample with 8 handles, for reasons that are not yet clear. We also probed whether the distribution of handles in the sample with 6 coiled-coils (e.g., on the edges vs clustered in the center of the cuboid) played any role, but observed only minor differences in the length distributions of these samples (Figures S13 and S14).

Based on these results, we concluded that the cuboids with 8 handles formed the most efficient arrays, so all of our subsequent experiments used this system. We next turned to a closer examination of the fibers with additional AFM images for the 8-handle system (Figure 5A-D). The individual cuboid structures could be readily distinguished, with small gaps between them where the peptides were attached. An AFM height profile (Figure $5 \mathrm{D}-\mathrm{F}$ ) showed a spacing of $\sim 40 \mathrm{~nm}$ between cuboids (which is close to the expected distance of $\sim 46 \mathrm{~nm}$ for the cuboid length $(32 \mathrm{~nm})$ and the intercuboid gap $(\sim 13 \mathrm{~nm}$, Figure $4 \mathrm{C})$ ) and a height of $12 \mathrm{~nm}$ across the fibers (which corresponds to a flattening of the actual height $(16 \mathrm{~nm})$, perhaps due to structure compression as a result of dehydration on the mica surface due to AFM imaging in air). Most of the fibers imaged were quite linear, with many consisting of $20-30$ monomers $(\sim 1 \mu \mathrm{m}$ in length). The longest fiber observed was composed of 49 cuboids and exceeded $2 \mu \mathrm{m}$. We attribute the extensive degree of supramolecular polymerization and the high persistence length of these structures to three key design features: (1) the multivalent nature of the coiled-coil interfaces, which promotes a "face-on" association to maximize peptide interactions; (2) the short linker between the DNA handles and the peptides compared with the much larger dimensions of the origami, which discourages peptides from creating branched junctions with three or more cuboids; and (3) the rigidity of the multihelical cuboid monomers, which prevents excessive bending. We also imaged the fibers formed in the 8 handle system by TEM (Figure $5 \mathrm{H}-\mathrm{K}$ ), and the results paralleled those obtained by AFM: long $1 \mathrm{D}$ arrays of cuboids linked at the interface bearing the peptides. In some images, the link between cuboids due to the peptide-DNA conjugates can be seen (Figure 5J, inset, yellow arrows). Measuring the length and width of the cuboids by TEM (Figure $5 \mathrm{~K}$, inset) gave dimensions of 36.8 and $19.7 \mathrm{~nm}$, respectively, which accurately reflect the design (Figure 4A). In both AFM and TEM images, a small fraction of intercuboid interfaces were not perfectly flush (Figure 5K, white arrows), perhaps due to incomplete linking of the two interfaces by the peptide-DNA conjugates.

In order to verify that the cuboid assembly was due to both the coiled-coil interaction between EI and $\mathrm{KI}$ and the DNA hybridization between $\mathrm{A} / \mathrm{A}^{*}$ and $\mathrm{B} / \mathrm{B}^{*}$, we performed a series 
A
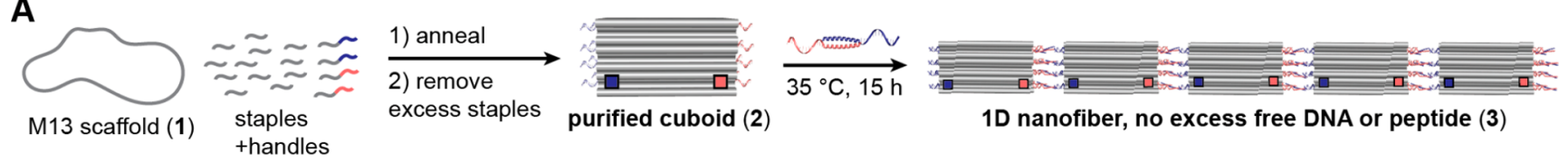

1D nanofiber, no excess free DNA or peptide (3)
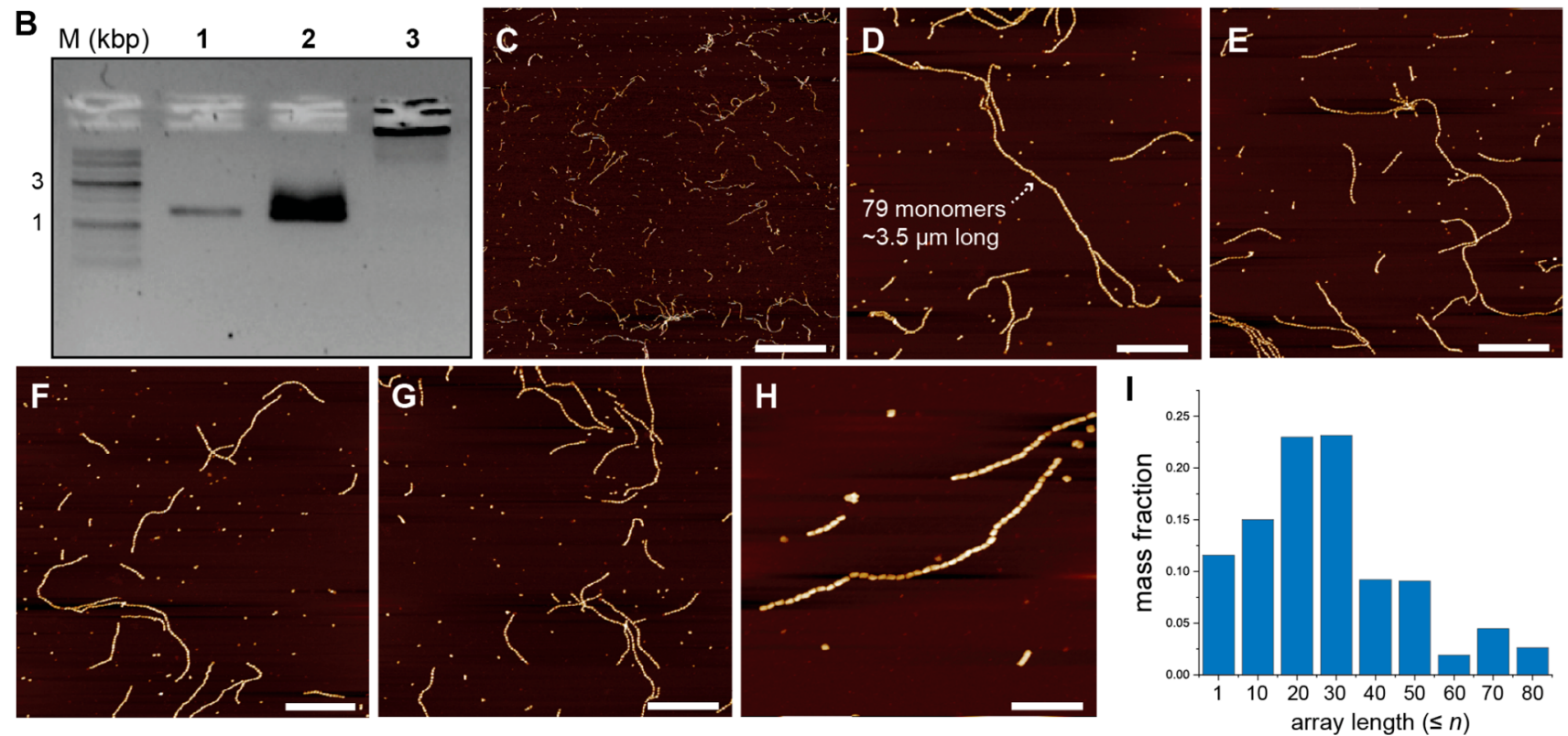

Figure 7. Hierarchical assembly of purified cuboids with coiled-coil DNA conjugates. (A) Schematic of assembly protocol. Pink and blue squares on the origami indicate the location of handles $\mathrm{A}^{*}$ and $\mathrm{B}^{*}$, respectively. (B) AGE analysis of assembly. Lane M: dsDNA ladder; 1: M13 scaffold; 2: cuboid with $A^{*}$ and $B^{*}$ following purification. Note the absence of a band for free staples; 3: cuboid with handles following incubation with preformed coiled-coil DNA conjugates. (C-H) AFM images of nanofibers formed after second incubation. Scale bars: $4 \mu \mathrm{m}(\mathrm{C}), 1 \mu \mathrm{m}(\mathrm{D}-\mathrm{G})$, $370 \mathrm{~nm}(\mathrm{H})$. (I) Histograms of mass fraction of origami for each array length for indicated samples. Numbers correspond to arrays between previous bin and indicated length, e.g., "30" denotes arrays $21-30$ cuboids in length.

of control experiments. Compared to the unmodified cuboid band by AGE (Figure 5G, lane 2 ), the addition of eight $A^{*}$ and $\mathrm{B}^{*}$ handles to each side of the cuboid did not significantly change the gel mobility (lane 3 ). Inclusion of A-EI and B-KI into the annealing mixture gave the expected aggregation band that did not enter the gel (lane 4). By contrast, cuboids annealed with A-EI and B-KI $\mathrm{scr}_{\text {(lane 5) or A-EI }}$ scr and B-KI (lane 6) did not show any aggregate bands, suggesting that the arrays are mediated by coiled-coil formation and not simply as a result of coating one side of the structure with a high density of cationic peptides. Finally, cuboids bearing $\mathrm{A}^{*}$ and $\mathrm{B}^{*}$ handles, but annealed with peptides linked to noncomplementary, poly $(\mathrm{T})$ strands $\left(\mathrm{T}_{14}\right.$-EI and $\left.\mathrm{T}_{14}-\mathrm{KI}\right)$ also did not show any aggregation in the loading well (lane 7), confirming that both self-assembly modes (coiled-coil formation and Watson-Crick pairing) are critical for hierarchical nanostructure formation. We also explored whether the linker length between the DNA handle and the coiled-coil peptides had any effect on the efficiency of assembly, reasoning that transitioning directly from the DNA to the peptide could result in higher binding efficiency and longer nanofibers. All the above experiments used DNA purchased from a commercial vendor and included a C6 alkyl linker between the 5 ' phosphate and the terminal amine, followed by an additional six carbons between the amine and the DBCO moiety (Figure S18A). To reduce this length, we synthesized a phosphoramidite with only two carbons and a cyclopropyl ring separating the $5^{\prime}$ phosphate and the cyclooctyne (Figure S18A), following a previous report. $^{29}$ This [6.1.0] bicyclononyne $(\mathrm{BCN})$ moiety was appended to the same DNA handles via solid-phase oligonucleotide synthesis to generate the shortened linker versions $A_{B C N}$ and $B_{B C N}$, and subsequently conjugated to the peptides to yield $A_{B C N}-E I$ and $B_{B C N}-K I$ (see section $S 8$ for characterization of $\mathrm{BCN}$ strands and peptide-DNA conjugates). The one-pot assembly of these conjugates with the cuboid origami yielded long $1 \mathrm{D}$ fibers that were morphologically indistinguishable from those with the DBCO-based conjugates (Figure S18E). We determined the length distribution of these fibers (Figure S18F) and the distribution was fairly similar to that of the DBCO-based arrays (Figure $4 \mathrm{~F}$ ), perhaps slightly shifted to shorter arrays. Indeed some flexibility between the peptide and the DNA handle may be beneficial to allow the coils to bind one another efficiently. Thus, for the next set of experiments we continued with the DBCO-based linkers.

Sequential, Hierarchical Assembly Pathways for Origami Arrays. One of our overarching goals in creating peptide-DNA nanostructures is to generate a toolkit of components that can be modularly assembled into more complex structures in a second step. Toward this end, we reasoned that if we separately modified individual cuboid blocks with only a single type of peptide (either at one or both ends), we could mix these structures in a second step and allow the free peptide "faces" to drive hierarchical assembly. We term the cuboids with only A-EI peptides on one side " $E_{c u b "}$ structures, the ones with only B-KI on one side " $K_{\mathrm{cub}}$ " structures, and the ones with $\mathrm{A}-\mathrm{EI}$ and $\mathrm{B}-\mathrm{KI}$ on both sides as " $E E_{\text {cub }}$ " and " $\mathrm{KK}_{\mathrm{cub}}$ " cuboids. Mixing $\mathrm{E}_{\mathrm{cub}}$ with $\mathrm{K}_{\mathrm{cub}}$ is expected 
to give a dimeric structure (Figure $6 \mathrm{~A}$ ), mixing $\mathrm{E}_{\text {cub }}$ with $\mathrm{KK}_{\mathrm{cub}}$ or $\mathrm{K}_{\text {cub }}$ with $\mathrm{EE}_{\mathrm{cub}}$ should give trimers (Figure $6 \mathrm{~B}, \mathrm{C}$ ), and mixing $\mathrm{EE}_{\text {cub }}$ with $\mathrm{KK}_{\text {cub }}$ should give copolymers with alternating cuboids (Figure 6D). Each individual modified cuboid was annealed as in the one-pot system, but components were then mixed in a second step and incubated at $32{ }^{\circ} \mathrm{C}$ for $15 \mathrm{~h}$. This lower-temperature protocol (optimized for assembly, vide infra) should allow the cuboids to assemble, without disrupting the core structure $\left(T_{\mathrm{m}} \sim 55^{\circ} \mathrm{C}\right.$ for a typical $3 \mathrm{D}$ origami structure).$^{30} \mathrm{We}$ first analyzed the individual building blocks by AGE (Figure $6 \mathrm{E}$ ). $\mathrm{E}_{\text {cub }}, \mathrm{K}_{\text {cub }}$, and $\mathrm{EE}_{\text {cub }}$ (lanes 2-4) all primarily showed a band corresponding to the monomeric cuboid structure. We note that some dimers and short oligomers form as well, due to nonspecific base stacking (which is commonly seen with DNA origami structures). This stacking was not observed in the one-pot system because the annealing ramp included a fast temperature decrease from 40 ${ }^{\circ} \mathrm{C}$, preventing these lower-energy interactions from forming; however, sequential assembly required a lower temperature incubation for the second step. The $\mathrm{KK}_{\text {cub }}$ structure, by contrast, showed a smeared band (lane 5), which we attribute to nonspecific aggregation of the structures resulting from the presence of two highly cationic surfaces. Unlike the arrays in Figure 5, many of these aggregates were not well-formed straight assemblies, but rather cuboids intersecting at various angles, perhaps due to the cationic surface of one cuboid binding to one of the other four exposed sides of another (Figure S23). We also note that the $\mathrm{KK}_{\text {cub }}$ smeared band is only seen after the second incubation step; all the initial, asformed cuboids do not show this aggregation (Figure 6E, inset lanes 11-14). Upon mixing $\mathrm{E}_{\text {cub }}$ with $\mathrm{K}_{\text {cub }}$ and incubating as described above a strong dimer band was seen in the gel (lane 6, yellow arrow), and both AFM (Figure 6F) and array length histograms (Figure 6G) confirmed that the dimer was the predominant structure. The fraction of dimers that formed as a function of handles yielded a sigmoidal curve (Figure S26), suggesting that peptide binding is cooperative. Likewise, combining $\mathrm{E}_{\text {cub }}$ with $\mathrm{KK}_{\text {cub }}$ (lane 7) or $\mathrm{K}_{\text {cub }}$ with $\mathrm{EE}_{\text {cub }}$ (lane 8) gave primarily a trimer band (red arrow), a result mirrored by array length histograms. Although the presence of nonspecific base stacking complicated the results, we note that purification of the desired structures (e.g., trimers) by gel extraction or gradient ultracentrifugation could circumvent this issue. Combining $\mathrm{EE}_{\text {cub }}$ with $\mathrm{KK}_{\text {cub }}$ yielded an intense band in the well by AGE (lane 9) and gave nanofibers similar to the one-pot system: relatively straight and with a face-on interface, as visualized by AFM (Figure 6F). These fibers consist of alternating $\mathrm{EE}_{\text {cub }}$ with $\mathrm{KK}_{\text {cub }}$ fibers, and could in the future be used to position different molecules. The length distribution of this system was similar to that of the one-pot system with 8 handles, with a slightly less pronounced tail of longer arrays. We probed the temperature dependence of this sequential assembly by AGE and found that the arrays formed best at an incubation temperature of $32{ }^{\circ} \mathrm{C}$ (Figure S19). Above this temperature, only monomers or short dimers were observed, and we estimated that the supramolecular association between $\mathrm{EE}_{\text {cub }}$ and $\mathrm{KK}_{\text {cub }}$ has an effective "melting temperature" for polymerization $\sim 34{ }^{\circ} \mathrm{C}$, though we cannot determine whether this is due to the exchange of the DNA hybridization or the coiled-coil assembly at the low (nM) concentrations used. Future work will probe the relative contribution of each supramolecular mode in driving hierarchical self-assembly.
Finally, we explored a second hierarchical assembly pathway: formation of cuboids bearing handles $A^{*}$ and $B^{*}$ (as in the one-pot system), followed by addition of preformed A-EI/B-KI coiled-coil and a second incubation at $35{ }^{\circ} \mathrm{C}$ for $15 \mathrm{~h}$ (Figure $7 A)$. In this case, the coiled-coil with DNA handles serves as a modular building block that can be assembled first and then added as a hybrid linker to the preassembled origami structures. To demonstrate the generality of our methodand to probe self-assembly in the absence of excess of free staples/peptides-we first purified the cuboids via spin filtration prior to addition of the peptides. We also only added an equimolar amount of peptide-DNA conjugates relative to the handles (i.e., 8 equiv relative to the cuboid with 8 handles). Analysis by AGE (Figure 7B) showed a clear monomer band for the cuboid with handles (lane 2), and the absence of any higher mobility band for free DNA or peptide, confirming that our purification protocol was successful. After subsequent incubation with the peptide-DNA conjugates, an intense band was seen in the loading well (lane 3), with no noticeable monomer or short oligomer bands/smear, suggesting an overall longer distribution of arrays. Indeed, a wide-field $\left(20 \times 20 \mu \mathrm{m}^{2}\right)$ AFM scan (Figure 7C) showed a large number of extremely long fibers, many approaching $3 \mu \mathrm{m}$ in length, with the longest observed consisting of 79 cuboids and reaching $\sim 3.5 \mu \mathrm{m}$ (Figure $7 \mathrm{D}-\mathrm{H}$ ). A histogram of the array length distribution (Figure 7I) paralleled the AFM results, with the largest number of fibers comprised of 20-30 cuboids, and a significant number from 30 to 80 monomers in length, far exceeding the longest fibers seen with the other two assembly methods. We compared this protocol to sequential arrays formed without intermediate purification of the cuboid and found that the length distribution was roughly similar (Figure S28). However, we note that the lack of purification in this sample could be offset by the 10-fold excess of peptide-DNA conjugates relative to the handles.

\section{CONCLUSIONS}

Our results are the first example of DNA nanostructures forming extended arrays through the self-assembly of coiledcoil interactions. We explored several different assembly pathways (one-pot vs two hierarchical protocols), created arrays and dimeric or trimeric structures, confirmed the specificity of both the coiled-coil and Watson-Crick selfassembly modes, and demonstrated that the self-assembly works both with and without intermediate purification steps. Assembling cuboids with preformed coiled-coil-DNA conjugates gave the longest arrays. Although we only focused on 1D arrays of DNA origami nanostructures in this report, our approach should be readily extensible to $2 \mathrm{D}$ arrays and perhaps even $3 \mathrm{D}$ crystals by designing the appropriate branched nanostructure monomers. In principle, our approach can also be extended to a wide range of coiled-coils, ${ }^{31}$ including larger homo- or hetero-oligomers. For example, a homotrimeric parallel coiled-coil could serve as a C3-symmetric "cap" for a DNA structure, similar to a recent report from our lab using homotrimeric proteins. ${ }^{10}$ Different coiled-coil systems have a wide range of stabilities, so it may be possible to sequentially form different domains during the annealing process, and effectively "fold" a DNA structure using orthogonal peptide interactions, akin to intramolecular coiled-coil folded cages. ${ }^{23}$ Using coils that are closer to charge-neutral, as described by Woolfson and Turberfield, ${ }^{24}$ could also help avoid some of the challenges with DNA conjugation and nonspecific aggregation. 
Several reports have showed that coil assembly can be controlled with light using azobenzenes, ${ }^{32}$ or that coils can be displaced similar to $\mathrm{DNA}^{8,9}$ by introducing an unpaired partial heptad "toehold"; ${ }^{33}$ both of these mechanisms can in principle now be used to dynamically control peptide-DNA structure self-assembly. Our ultimate goal is to build nanomaterials that rival the complexity of DNA origami, but contain multiple unique peptide structure units, enabling truly hybrid peptide-DNA nanostructures possessing added functionalities and greater chemical diversity.

\section{ASSOCIATED CONTENT}

\section{S Supporting Information}

The Supporting Information is available free of charge at https://pubs.acs.org/doi/10.1021/jacs.9b11158.

Synthetic protocols and characterization of all peptide and peptide-DNA conjugates, design and strand sequences of all DNA nanostructures (DX tiles and origami), additional AFM characterization and unbinned histograms of all systems probed, comparison of BCNlinker system (PDF)

\section{AUTHOR INFORMATION}

\section{Corresponding Author}

*nstepha1@asu.edu

\section{ORCID $\odot$}

Alex Buchberger: 0000-0003-3412-8945

Chad R. Simmons: 0000-0002-2290-6132

Ronit Freeman: 0000-0001-5960-6689

Nicholas Stephanopoulos: 0000-0001-7859-410X

\section{Notes}

The authors declare no competing financial interest.

\section{ACKNOWLEDGMENTS}

The authors would like to thank the H. Yan lab for use of their AFM instrument. The authors would like to thank Raghu Pradeep Narayanan for discussions on the DNA origami design and characterization, help with TEM imaging, and providing the M13 scaffold. N.S. acknowledges startup funds from Arizona State University. This material is based upon work supported by the Air Force Office of Scientific Research under Award Number FA9550-17-1-0053.

\section{REFERENCES}

(1) Rothemund, P. W. K. Folding DNA to create nanoscale shapes and patterns. Nature 2006, 440, 297-302.

(2) Douglas, S. M.; Dietz, H.; Liedl, T.; Hoegberg, B.; Graf, F.; Shih, W. M. Self-assembly of DNA into nanoscale three-dimensional shapes. Nature 2009, 459, 414-418.

(3) Hong, F.; Zhang, F.; Liu, Y.; Yan, H. DNA Origami: Scaffolds for Creating Higher Order Structures. Chem. Rev. 2017, 117 (20), 12584-12640.

(4) Winfree, E.; Liu, F. R.; Wenzler, L. A.; Seeman, N. C. Design and self-assembly of two-dimensional DNA crystals. Nature 1998, 394, 539-544.

(5) Yan, H.; Park, S. H.; Finkelstein, G.; Reif, J. H.; LaBean, T. H. DNA-templated self-assembly of protein arrays and highly conductive nanowires. Science 2003, 301, 1882-1884.

(6) Ke, Y.; Ong, L. L.; Shih, W. M.; Yin, P. Three-Dimensional Structures Self-Assembled from DNA Bricks. Science 2012, 338, $1177-1183$.

(7) Wei, B.; Dai, M.; Yin, P. Complex shapes self-assembled from single-stranded DNA tiles. Nature 2012, 485, 623-626.
(8) Zhang, D. Y.; Seelig, G. Dynamic DNA nanotechnology using strand-displacement reactions. Nat. Chem. 2011, 3, 103-113.

(9) Simmel, F. C.; Yurke, B.; Singh, H. R. Principles and Applications of Nucleic Acid Strand Displacement Reactions. Chem. Rev. 2019, 119, 6326-6369.

(10) Xu, Y.; Jiang, S. X.; Simmons, C. R.; Narayanan, R. P.; Zhang, F.; Aziz, A. M.; Yan, H.; Stephanopoulos, N. Tunable Nanoscale Cages from Self-Assembling DNA and Protein Building Blocks. ACS Nano 2019, 13, 3545-3554.

(11) Kashiwagi, D.; Sim, S.; Niwa, T.; Taguchi, H.; Aida, T. Protein Nanotube Selectively Cleavable with DNA: Supramolecular Polymerization of "DNA-Appended Molecular Chaperones. J. Am. Chem. Soc. 2018, 140, 26-29.

(12) McMillan, J. R.; Mirkin, C. A. DNA-Functionalized, Bivalent Proteins. J. Am. Chem. Soc. 2018, 140, 6776-6779.

(13) Brodin, J. D.; Auyeung, E.; Mirkin, C. A. DNA-mediated engineering of multicomponent enzyme crystals. Proc. Natl. Acad. Sci. U. S. A. 2015, 112, 4564-4569.

(14) Jiang, T.; Meyer, T. A.; Modlin, C.; Zuo, X. B.; Conticello, V. P.; Ke, Y. G. Structurally Ordered Nanowire Formation from CoAssembly of DNA Origami and Collagen-Mimetic Peptides. J. Am. Chem. Soc. 2017, 139, 14025-14028.

(15) Freeman, R.; Han, M.; Alvarez, Z.; Lewis, J. A.; Wester, J. R.; Stephanopoulos, N.; McClendon, M. T.; Lynsky, C.; Godbe, J. M.; Sangji, H.; Luijten, E.; Stupp, S. I. Reversible self-assembly of superstructured networks. Science 2018, 362, 808-813.

(16) Daly, M. L.; Gao, Y.; Freeman, R. Encoding Reversible Hierarchical Structures with Supramolecular Peptide-DNA Materials. Bioconjugate Chem. 2019, 30, 1864-1869.

(17) Apostolovic, B.; Danial, M.; Klok, H. A. Coiled coils: attractive protein folding motifs for the fabrication of self-assembled, responsive and bioactive materials. Chem. Soc. Rev. 2010, 39, 3541-3575.

(18) Hahn, J.; Wickham, S. F. J.; Shih, W. M.; Perrault, S. D. Addressing the Instability of DNA Nanostructures in Tissue Culture. ACS Nano 2014, 8, 8765-8775.

(19) Pandya, M. J.; Spooner, G. M.; Sunde, M.; Thorpe, J. R.; Rodger, A.; Woolfson, D. N. Sticky-end assembly of a designed peptide fiber provides insight into protein fibrillogenesis. Biochemistry 2000, 39, 8728-8734.

(20) Dong, H.; Paramonov, S. E.; Hartgerink, J. D. Self-assembly of alpha-helical coiled coil nanofibers. J. Am. Chem. Soc. 2008, 130, 13691-13695.

(21) Banwell, E. F.; Abelardo, E. S.; Adams, D. J.; Birchall, M. A.; Corrigan, A.; Donald, A. M.; Kirkland, M.; Serpell, L. C.; Butler, M. F.; Woolfson, D. N. Rational design and application of responsive alpha-helical peptide hydrogels. Nat. Mater. 2009, 8, 596-600.

(22) Fletcher, J. M.; Harniman, R. L.; Barnes, F. R. H.; Boyle, A. L.; Collins, A.; Mantell, J.; Sharp, T. H.; Antognozzi, M.; Booth, P. J.; Linden, N.; Miles, M. J.; Sessions, R. B.; Verkade, P.; Woolfson, D. N. Self-Assembling Cages from Coiled-Coil Peptide Modules. Science 2013, 340, 595-599.

(23) Gradisar, H.; Bozic, S.; Doles, T.; Vengust, D.; HafnerBratkovic, I.; Mertelj, A.; Webb, B.; Sali, A.; Klavzar, S.; Jerala, R. Design of a single-chain polypeptide tetrahedron assembled from coiled-coil segments. Nat. Chem. Biol. 2013, 9, 362-366.

(24) Lou, C. G.; Martos-Maldonado, M. C.; Madsen, C. S.; Thomsen, R. P.; Midtgaard, S. R.; Christensen, N. J.; Kjems, J.; Thulstrup, P. W.; Wengel, J.; Jensen, K. J. Peptide-oligonucleotide conjugates as nanoscale building blocks for assembly of an artificial three-helix protein mimic. Nat. Commun. 2016, 7, 12294.

(25) Jin, J.; Baker, E.; Wood, C.; Bath, J.; Woolfson, D.; Turberfield, A. Peptide Assembly Directed and Quantified Using Megadalton DNA Nanostructures. ACS Nano 2019, 13, 9927-9935.

(26) Aronsson, C.; Danmark, S.; Zhou, F.; Oberg, P.; Enander, K.; $\mathrm{Su}$, H.; Aili, D. Self-sorting heterodimeric coiled coil peptides with defined and tuneable self-assembly properties. Sci. Rep. 2015, 5, 14063.

(27) Stephanopoulos, N.; Freeman, R.; North, H. A.; Sur, S.; Jeong, S. J.; Tantakitti, F.; Kessler, J. A.; Stupp, S. I. Bioactive DNA-Peptide 
Nanotubes Enhance the Differentiation of Neural Stem Cells Into Neurons. Nano Lett. 2015, 15, 603-609.

(28) Tigges, T.; Heuser, T.; Tiwari, R.; Walther, A. 3D DNA Origami Cuboids as Monodisperse Patchy Nanoparticles for Switchable Hierarchical Self-Assembly. Nano Lett. 2016, 16, 7870-7874.

(29) Jawalekar, A. M.; Malik, S.; Verkade, J. M. M.; Gibson, B.; Barta, N. S.; Hodges, J. C.; Rowan, A.; van Delft, F. L. Oligonucleotide Tagging for Copper-Free Click Conjugation. Molecules 2013, 18, 7346-7363.

(30) Sobczak, J. P. J.; Martin, T. G.; Gerling, T.; Dietz, H. Rapid Folding of DNA into Nanoscale Shapes at Constant Temperature. Science 2012, 338, 1458-1461.

(31) Wood, C. W.; Woolfson, D. N. CCBuilder 2.0: Powerful and accessible coiled-coil modeling. Protein Sci. 2018, 27, 103-111.

(32) Woolley, G. A.; Jaikaran, A. S. I.; Berezovski, M.; Calarco, J. P.; Krylov, S. N.; Smart, O. S.; Kumita, J. R. Reversible photocontrol of DNA binding by a designed GCN4-bZIP protein. Biochemistry 2006, $45,6075-6084$.

(33) Groger, K.; Gavins, G.; Seitz, O. Strand Displacement in Coiled-Coil Structures: Controlled Induction and Reversal of Proximity. Angew. Chem., Int. Ed. 2017, 56, 14217-14221. 